\title{
METODOLOGÍA PARA LA CARACTERIZACIÓN DE VARIABLES DE MARCHA HUMANA, MEDIANTE SISTEMA DE REGISTRO SUB ACUÁTICO: TOBILLO
}

\section{RESUMEN}

Mediante un sistema robótico que consta de un sistema de cadena y una plataforma móvil en el piso del tanque de marcha, que ajusta la posición final de la cámara submarina, se realizó análisis de la marcha y posterior cálculo de los ángulos articulares en ambiente acuático de 4 individuos voluntarios. El ángulo filtrado de flexión plantar del tobillo durante la fase de despegue del pie en el ciclo de marcha subacuática es mayor en el género femenino $(122.7 \pm 1.1)$ que en el masculino (118.4 \pm 2.5$)$. El ángulo filtrado de dorsiflexión del tobillo durante la fase de apoyo en el ciclo de marcha subacuática presenta "ligeras" diferencias, siendo mayor en el género masculino $(85.2 \pm 2.5)$ que en el femenino $(85.0 \pm 1.0)$. En conclusión, se pueden cuantificar las variables cinemáticas de la marcha humana (parámetros angulares del tobillo), en individuos sanos, usando video fotogrametría como sistema computarizado de captura de imágenes subacuáticas, en el tanque de marcha.

Palabras clave: marcha, tobillo, movimiento.

\section{METHODOLOGY FOR CHARACTERIZATION OF HUMAN GAIT VARIABLES USING AN UNDERWATER REGISTER SYSTEM: ANKLE}

\section{ABSTRACT}

Using a robotic system which is made of a chain and mobile platform system on the floor of gait tank, which adjusts the final position of a sub-aquatic camera, it was possible to carry out gait analysis and subsequent calculation of joint angles in an aquatic environment of four voluntary persons. The filtrated plantar flexion angle of the ankle during the rocking phase of the sub-aquatic gait cycle is higher in females $(122.7 \pm 1.1)$ than in males $(118.4 \pm 2.5)$. The filtered dorsiflexion angle of the ankle during the stance phase of the sub-aquatic gait cycle shows slight differences being higher in men $(85.2 \pm 2.5)$ than in females $(85.0 \pm 1.0)$. In conclusion, it is possible to quantify kinematic variables of human gait (angular ankle parameters) in healthy individuals using videophotogrammetry as a computerized system for capturing sub-aquatic images in the gait tank.

Key words: gait, ankle, motion.

\footnotetext{
${ }^{1}$ Centro de Rehabilitación Integral Teletón. Manizales, Colombia. E-mail: mauriciohernandovalencia@gmail.com (D) ORCID: 0000-0001-7294-6872

2Departamento de Ciencias Básicas de la Salud, Universidad de Caldas. Manizales, Colombia.

${ }^{3} \mathrm{Facultad}$ de Ciencias para la Salud. Universidad de Manizales. Manizales, Colombia. jose.osorio_o@ucaldas.edu.co (iD) ORCID: 0000-0002-6875-3215
} 


\section{INTRODUCCIÓN}

La hidroterapia es utilizada en los procesos de rehabilitación de numerosas patologías neurológicas y osteomusculares. La terapia acuática ha sido utilizada en los procesos de rehabilitación de personas con trastornos de la marcha desde hace muchos años, y el uso del tanque de marcha es frecuente como herramienta de trabajo, sin embargo existe poca evidencia documentada sobre la evaluación objetiva de la eficacia que tiene esta terapia en la recuperación de los pacientes (1).

En Colombia, han sido pocas las investigaciones realizadas sobre el estudio de variables biomecánicas cuantitativas que permitan establecer la eficacia real y sustentada de los tratamientos realizados con hidroterapia. Se ha diseñado un equipo robótico de captura de imágenes subacuáticas que permite medir los parámetros angulares de la rodilla y tobillo al deambular en el tanque de marcha; por lo cual se considera necesario cuantificar parámetros angulares y lineales de la marcha humana subacuática, estableciendo un modelo de mediciones y patrones estadísticos, que sirvan como punto de referencia para futuras investigaciones en el tanque de marcha.

\section{METODOLOGÍA}

El presente estudio fue realizado en el tanque de marcha del área de hidroterapia de la Clínica Universidad de la Sabana, en el municipio de Chía, departamento de Cundinamarca, Colombia, durante el mes de mayo de 2012. Es un estudio descriptivo en el que participaron 4 individuos voluntarios ( 2 mujeres y 2 hombres) mayores de 18 años, quienes firmaron libremente el consentimiento informado. Con base en el artículo 5 del capítulo 1 de la Resolución 8430 del 4 de octubre de 1993 del Ministerio de Salud de Colombia (actualmente Ministerio de la Protección Social) sobre los aspectos éticos de la investigación en seres humanos, en este trabajo prevalece el criterio del respeto a la dignidad, protección de los derechos y el bienestar humano. Este trabajo se clasifica como investigación con riesgo mínimo que emplea el registro de datos a través de dos procedimientos comunes: una fase inicial de examen físico general y toma de medidas angulares de miembros inferiores a través de un goniómetro, y una segunda fase en la que se aplican unas marcas autoadhesivas en los miembros inferiores del individuo, que luego es filmado al hacerlo pasar caminando por un pasillo sumergido en agua hasta el nivel del apéndice xifoides. El proceso estuvo monitorizado por el grupo de monitoria clínica de la Universidad de La Sabana para garantía de la calidad de la información y el cumplimiento de las normas de buenas prácticas clínicas. Los criterios de inclusión del presente trabajo fueron individuos adultos jóvenes sanos, mayores de 18 años, que participaron de manera voluntaria y firmaron el consentimiento informado. Los criterios de exclusión fueron las contraindicaciones relativas y absolutas para permanecer en ambiente acuático previstas por la literatura, a saber: presencia de heridas abiertas, procesos infecciosos o inflamatorios agudos, hipertensión arterial no controlada, alteraciones cardíacas o pulmonares en períodos no estables, insuficiencias orgánicas en períodos de descompensación (2), mal estado general, pacientes desorientados, epilepsia no controlada, diabetes no controlada y tuberculosis (3). Personas con alteraciones del patrón de marcha, con limitaciones físicas de los sistemas óseo, muscular, articular, nervioso y cardiopulmonar diagnosticados (4). Para la recolección de los datos, los sujetos fueron sometidos inicialmente a una selección denominada fase I, a través de una entrevista y un examen físico general. Los sujetos seleccionados fueron sometidos a un análisis clínico observacional de la marcha en ambiente terrestre, por el médico residente de fisiatría en la fase II. Los sujetos seleccionados fueron sometidos a un análisis cinemático de la marcha en ambiente acuático en la fase III. Para la selección de los sujetos se utilizó una ficha de evaluación elaborada para el estudio. 
Se analizaron 4 individuos adultos sanos $\sin$ antecedentes patológicos así:

Individuo 1: género femenino, edad 21 años, dominancia cerebral izquierda, peso de $58 \mathrm{~kg}$, talla $1,70 \mathrm{~m}$, IMC: $20,06 \mathrm{Kg} / \mathrm{m}^{2}$ con signos vitales estables (T/ A 110/70 mm de Hg brazo derecho sentado y 110/70 mm de $\mathrm{Hg}$ brazo derecho en bípedo, Frecuencia cardiaca $76 \times$ minuto, Frecuencia respiratoria: $16 \times$ minuto).

Individuo 2: género femenino, edad 30 años, dominancia cerebral izquierda, peso de $65 \mathrm{~kg}$, talla 1,68 m, IMC: $23,04 \mathrm{Kg} / \mathrm{m}^{2}$ con signos vitales estables (T/A 120/70 mm de Hg brazo derecho sentado y 115/70 mm de Hg brazo derecho en bípedo, Frecuencia cardiaca $72 \times$ minuto, Frecuencia respiratoria: $16 \times$ minuto).

Individuo 3: género masculino, edad 26 años, dominancia cerebral derecha, peso de $72 \mathrm{~kg}$, talla 1,70 m, IMC: $24,91 \mathrm{Kg} / \mathrm{m}^{2}$ con signos vitales estables (T/A 110/80 mm de Hg brazo derecho sentado y $110 / 70 \mathrm{~mm}$ de $\mathrm{Hg}$ brazo derecho en bípedo, Frecuencia cardiaca 70 x minuto, Frecuencia respiratoria: $18 \times$ minuto).

Individuo 4: género masculino, edad 38 años, dominancia cerebral izquierda, peso de $82 \mathrm{~kg}$, talla 1,76 m, IMC: $26,47 \mathrm{Kg} / \mathrm{m}^{2}$ con signos vitales estables (T/A 130/80 mm de Hg brazo derecho sentado y 130/80 mm de $\mathrm{Hg}$ brazo derecho en bípedo, Frecuencia cardiaca $80 \times$ minuto, Frecuencia respiratoria: $18 \times$ minuto). Para la evaluación de la fuerza muscular se utilizó la escala de fuerza muscular del Medical Research Council del Reino Unido y los 4 individuos examinados tuvieron una fuerza muscular de 5/5 en los miotomas examinados.

Análisis de la marcha y posterior cálculo de los ángulos articulares en ambiente acuático: se marcaron puntos anatómicos en el miembro inferior derecho de cada individuo a través de marcadores pasivos que se ubicaron de acuerdo con el modelo bidimensional de cuatro segmentos (5). El miembro inferior fue dividido en tres segmentos: cadera, pierna y pie. Los segmentos fueron delimitados por puntos localizados en estructuras anatómicas: cóndilo femoral lateral y maléolo lateral; los puntos fueron referenciados en un mismo sistema de coordenadas (6). Los marcadores utilizados fueron circulares de $3,5 \mathrm{~cm}$ de diámetro, de color amarillo con borde negro, impermeables, que se fijaron a las superficies articulares y puntos de referencia con velcro. Los 4 individuos fueron sumergidos hasta el nivel de los apéndices xifoides y posteriormente analizados de manera individual en el tanque de marcha. Para la evaluación de los ángulos de movimiento, se filmó al sujeto en movimiento en el plano sagital, se le pidió que caminara a una velocidad constante y cómoda para él, a través del tanque de marcha, donde se ubicó un eje de robot que permitió el movimiento horizontal y vertical de una cámara subacuática adjunta, y que realizó la adquisición de la secuencia de imágenes, que posteriormente se analizaron para el cálculo del ángulo del tobillo y su variación durante las fases y subfases de la marcha subacuática. En cada individuo se realizaron 5 secuencias de filmación del tobillo derecho durante cuatro pasos en el tanque de marcha.

Sistema robótico: consta de un sistema de cadena y una plataforma móvil en el piso del tanque, que ajusta la posición final de la cámara submarina. El equipo tiene 4 metros de longitud $y$ un ancho de 1,5 metros, hay un espacio de $15 \mathrm{~cm}$ de ancho entre la pared de la piscina y barras de soporte lateral, la cámara es compatible con una plataforma sobre el robot. Su ubicación está entre la pared de la piscina y barras de soporte lateral; para el movimiento de la cámara de video se utiliza un sistema de cadena y tren que arrastra la cadena en sus enlaces. La herramienta desarrollada está constituida por un eje robótico que permite el movimiento horizontal y vertical de una cámara subacuática acoplada a este; el sistema realiza el seguimiento de la persona dentro del tanque de marcha y la adquisición de la secuencia de imágenes que posteriormente se procesa para obtener el cálculo 
del ángulo del tobillo, así como su variación en el tiempo. El sistema mecánico del eje robótico está compuesto por un actuador (motor), un sistema de transmisión por engranaje - cadena y una plataforma móvil en el piso del tanque que ajusta la posición final de la cámara subacuática. El sistema es controlado electrónicamente con una palanca de mando.

El controlador está basado en un micro controlador comercial y el sistema de visión consiste en un casco cámara análogo submarino, con un objetivo de $2,6 \mathrm{~mm}$, con 120 grados de alcance angular óptico, que tiene una cámara con una resolución de 240.480 Pixeles, es decir una resolución de 112.5 kilo pixeles y con una velocidad de la cámara de 30 cuadros por segundo. El voltaje de alimentación es de $12 \mathrm{~V}$. La captura y digitalización de la secuencia de imágenes se realiza a través de un importador de tarjeta de vídeo Pinnacle, que transmite la información a un computador portátil mediante el puerto USB. El almacenamiento de información de vídeo en formato AVI es inicialmente en el equipo y, a continuación, en una unidad externa y portable.

Procesamiento del video y cálculo del ángulo del tobillo: lo realiza un software con lenguaje C\#net. Se utilizaron como bibliotecas adicionales para el tratamiento de la imagen el Aforge. Net (http://www.aforgenet.com/). El sistema permite grabar en video lo que se adquiere por la cámara, y a partir de estos videos, se puede hacer análisis del ángulo entre 3 marcadores, ya sea de manera manual o automática, y permite revisar los videos grabados cuadro por cuadro. Se diseñó un algoritmo de procesamiento para el protocolo de adquisición de imágenes. Este algoritmo se basa en un método automático para el cálculo de la variación del ángulo del tobillo, que consiste en una etapa de pre procesamiento de la secuencia de imágenes, otra de segmentación de los marcadores pasivos del eje central de la pierna, seguido del procesamiento basado en morfología matemática, y finalmente la etapa de cálculo del ángulo.
Manejo de datos: fueron utilizados Microsoft Excel y Minitab Statistical Software.

\section{RESULTADOS}

Se evaluaron 10 ciclos de marcha sobre la banda por persona como número de ciclos válidos; sumergidos hasta el nivel del apéndice xifoides, y 5 capturas por sesión y movimiento para cada uno, con recorrido de 4 metros, durante 10 segundos, con velocidad de paso determinado por condiciones de 'comodidad' otorgadas por cada individuo para las cinco pruebas; con desentrelazado de 30 fotogramas por segundo, logrando la digitalización de marcadores superficiales con mayor precisión por medio del sistema utilizado; exportando datos cinemáticos (duración del paso, rangos de movimiento por segmentos corporales) en proyección sobre el plano sagital para tres pasos con longitud completa.

Duración de paso: se entiende como longitud de paso completo, la distancia lineal entre los sucesivos puntos de contacto del talón del mismo pie; la duración de paso será entendida entonces, como el tiempo que el individuo tarde en ejecutar esta acción (7). Se tomó como muestra, la duración de paso completa (DPC) de tres recorridos en 5 pruebas, con criterio de velocidad 'comodidad' otorgada por cada sujeto, encontrando tiempo en segundos. Para estas condiciones, se encontraron duraciones de paso en segundos, con valores mínimos (media 1.81) y máximos (media 4.16) en el género femenino para realizar un paso completo en condiciones subacuáticas; por otra parte, valores medianos 'aproximados' en el masculino (media $2.64-2.24)$. El $45 \%$ de los datos obtenidos para tobillo cuentan con confiabilidad estadística representada con $\mathrm{P}<0.05$.

Comportamiento del tobillo en individuo femenino 1, ángulo filtrado Datos estadísticamente confiables para el $40 \%$ de las pruebas (Prueba 4-5): P< 0,05: el rango de movimiento para la flexión de tobillo de 
ángulo filtrado en fase de despegue del pie en grados, cuenta con una media de 138.2, con valor mínimo de 105.7 en prueba 1 paso $2 \mathrm{y}$ máximo de 149.32 en prueba 5 paso 3 (tabla 1). Existe un patrón predominante de tipo ascendente en ángulos de flexión plantar durante la progresión de la marcha prueba a prueba. El rango de movimiento para la flexión de tobillo de ángulo filtrado en fase de apoyo plantar en grados, cuenta con una media de 87.71 , con valor mínimo de 77.88 en la prueba 4 paso 3 y máximo de 98.63 en prueba 2 paso 1 (tabla 2 ).

\section{Comportamiento del tobillo en individuo} femenino 2 - ángulo filtrado. Datos estadísticamente confiables para el $80 \%$ de las pruebas (Prueba 2-5): $\boldsymbol{P}<0$,05: el rango de movimiento para la flexión de tobillo de ángulo filtrado en fase de despegue del pie en grados, cuenta con una media de 130.4 , con valor mínimo de 105.07 en prueba 1 paso $2 \mathrm{y}$ máximo de 142.19 en prueba 3 paso 3 (tabla 3 ). Existe un patrón predominante de tipo ascendente en ángulos de flexión plantar durante la progresión de la marcha prueba a prueba. El rango de movimiento para la flexión de tobillo de ángulo filtrado en fase de apoyo plantar en grados, cuenta con una media de 84.84 , con valor mínimo de 78.46 en la prueba 2-3 paso 2-3 y máximo de 91.15 en prueba 2 paso 3 (tabla 4).

Comportamiento del tobillo en individuo masculino 1 - ángulo filtrado. Datos estadísticamente confiables para el $40 \%$ de las pruebas (Prueba 1-3). P< 0,05: el rango de movimiento para la flexión de tobillo de ángulo filtrado en fase de despegue del pie en grados, cuenta con una media de 118.67 , con valor mínimo de 103.3 en prueba 4 paso 1 y máximo de 140.35 en prueba 1 paso 3 (tabla 5). El rango de movimiento para la flexión de tobillo de ángulo filtrado en fase de apoyo plantar en grados, cuenta con una media de 80.73 , con valor mínimo de 60.00 en la prueba 3 paso 3 y máximo de 92.29 en prueba 1 paso 3 (tabla 6).
Comportamiento del tobillo en individuo masculino 2 - ángulo filtrado. Datos estadisticamente confiables para el $20 \%$ de las pruebas (Pruebas 1-5). $\quad P<0,05$ : el rango de movimiento para la flexión de tobillo de ángulo filtrado en fase de despegue del pie en grados, cuenta con una media de 121.4, con valor mínimo de 102.7 en prueba 1-5 paso 3 y máximo de 135.23 en prueba 5 paso 2 (tabla 7). Existe un patrón de estabilización gradual en ángulos de flexión plantar durante la progresión de la marcha prueba a prueba. El rango de movimiento para la flexión de tobillo de ángulo filtrado en fase de apoyo plantar en grados, cuenta con una media de 81.73, con valor mínimo de 76.11 en la prueba 4 paso 3 y máximo de 92.87 en prueba 3 paso 2 (tabla 8).

\section{DISCUSIÓN}

Teniendo en cuenta que la terapia física y los procesos de rehabilitación deben tener un punto de apoyo en datos mensurables y observacionales objetivos, y que hay pocos estudios que vinculen variables biomecánicas en el medio acuático; el presente estudio analiza el movimiento de personas sanas en el medio acuático a través de los parámetros cinemáticos angulares de tobillo en las fases y subfases de la marcha, usando un sistema electrónico que permite medir variables biomecánicas, a partir de un sistema de imágenes digitalizadas adquiridas en el tanque de marcha. En el año 2005 se evaluaron las características de la marcha a diferentes velocidades en tierra y en agua, en 16 individuos adultos jóvenes sanos describiendo la actividad electromiográfica del Bíceps femoris y del Gluteus maximus, y haciendo mediciones cinemáticas y cinéticas (8). Utilizaron cinco marcadores en el lado derecho del tronco y la extremidad inferior derecha a nivel del centro geométrico de las articulaciones, ubicándolos en el punto medio de la cresta iliaca entre la espina iliaca anterosuperior y posterosuperior, el trocánter mayor, el cóndilo femoral lateral, el maléolo lateral y la quinta articulación metatarsofalángica. 
Concluyeron que el grupo muscular del tobillo está encargado de soportar el peso contra la gravedad y que el grupo muscular involucrado en la extensión de la cadera contribuye con la propulsión de la marcha. Además que durante la marcha en agua se reducen los momentos articulares y cambia completamente la coordinación interarticular (8); lo cual fue confirmado en el presente estudio. Al analizar los parámetros temporales y de los componentes verticales de la fuerza de reacción del suelo (FRS) al andar en medios acuáticos a una profundidad de la espina-iliaca-antero-superior, el valor medio de pico máximo se encuentra entre 60 y $70 \%$. Los resultados muestran una disminución de aproximadamente la mitad en el componente vertical de la FRS y un aumento de los valores temporales cuando son comparados con la marcha en el suelo (9). El ángulo del tobillo tiene más flexión plantar en agua durante la fase de apoyo y al final de la fase de balanceo que en tierra (10). En la fase de apoyo inicial en tierra, los individuos realizan durante el apoyo del calcáneo, dorsiflexión del tobillo que disminuye a la posición neutra articular en la fase de apoyo medio. En el apoyo final la articulación está en plantiflexión y a medida que la fase de oscilación ocurre, la articulación pasa nuevamente a una posición neutra hacia el balanceo final, donde nuevamente estará en dorsiflexión (11). Algunos autores (12) aclaran que la articulación del tobillo en ambiente acuático está prácticamente neutra durante el apoyo inicial. Otros muestran un pico de dorsiflexión en el apoyo medio para el ambiente acuático (13), mientras que otros aclaran cualitativamente que el tobillo presenta en el ambiente acuático una disminución de la dorsiflexión en el apoyo inicial (14). Estos resultados son diferentes a los encontrados en otros estudios, donde a partir del apoyo medio y hasta el apoyo final reportan una variación angular mayor del tobillo en dirección a la plantiflexión la cual aumenta significativamente en este periodo $(15,16)$. Ha sido aclarado que durante el $60 \%$ de la fase de apoyo y en la oscilación final, la plantiflexión del tobillo en agua es mayor (10). Estudios anteriores constatan que la actividad eléctrica del músculo tibial anterior, músculo responsable de la dorsiflexión, es menor en ambiente acuático $(8,17)$. Puede deducirse entonces, que los sujetos en ambiente acuático buscarán mayor contacto con la superficie para garantizar mayor estabilidad y equilibrio. Por lo tanto, en el medio acuático se presentan diferencias significativas en relación con el comportamiento articular en sus fases y subfases, en comparación con la marcha en ambiente terrestre, que deben ser consideradas durante la elaboración de un programa de tratamiento que utilice la hidroterapia como recurso. En el medio acuático la articulación del tobillo realiza apoyo en el talón, permaneciendo en una posición neutra principalmente durante la fase de apoyo inicial. Estas diferencias pueden atribuirse al hecho de que el cuerpo humano adopta patrones de movimiento y diferentes estrategias motoras mientras camina en el medio acuático. Esto ocurre probablemente, por la necesidad de adaptar el sistema sensoriomotriz al deambular en un medio con diferentes características físicas. La falta de familiaridad y las condiciones ambientales impuestas por el medio líquido promueven adaptaciones al movimiento. Se deben tener en cuenta algunos factores como la edad, ya que al examinar los parámetros cinemáticos y cinéticos de la marcha en personas ancianas sanas (70 \pm 6 años) caminando en agua y en tierra comparados con un grupo de referencia de adultos jóvenes ( $29 \pm 6$ años), se ha encontrado que las personas más viejas, al caminar en tierra, tienen menor longitud de zancada, velocidad más lenta del paso, valores más bajos de la fuerza de reacción al piso, impulsos horizontales superiores, rango menor del movimiento de la rodilla y dorsiflexión menor del tobillo (11). En el ámbito nacional, el programa de ingeniería biomédica de la Escuela de Ingeniería de Antioquía y la Universidad CES Medellín, trabaja sobre las consideraciones para el análisis de la marcha humana, con técnicas de videogrametría, electromiografía y dinamometría, por ser estos los de mayor utilización en la actualidad, mostrando los parámetros espaciotemporales, cinéticos y cinemáticos que usualmente se analizan en 
estudios de marcha y detallan las consideraciones para la configuración y proceso de adquisición de un laboratorio de marcha (18). En el presente estudio, el sistema robótico y el software utilizados son sensibles para cuantificar las variables cinemáticas de la marcha humana, en individuos sanos, a diferentes velocidades de paso completo en el tanque de marcha. Se recomienda utilizar marcadores adheridos a la piel en puntos anatómicos de referencia como se menciona en el protocolo Davis, para evitar desplazamientos independientes. El pasillo de $4 \mathrm{~m}$ es suficiente para realizar 2 ciclos completos, y el análisis debe realizarse sobre los dos pasos intermedios (1 sólo ciclo), de modo que no se incluyan el paso inicial ni el final. Se debe adaptar un sistema de banda en el piso que permita una velocidad constante de paso completo para realizar una toma de video efectiva desde el primer paso.

\section{CONCLUSIONES}

Según resultados obtenidos, aportamos valores de cuantificación de las variables cinemáticas de la marcha humana (tobillo), en individuos sanos, usando video fotogrametría como sistema computarizado de captura de imágenes subacuáticas, en el tanque de marcha. Se debe tener en cuenta que se trabajó con individuos normales y no con pacientes que presenten deformidades del pie o rotaciones importantes en el plano transverso, en cuyo caso, debería hacerse otro trabajo de investigación utilizando la metodología aquí propuesta.

\section{REFERENCIAS}

1. Al-Qubaeissy KY, Fatoye FA, Goodwin PC, Yohannes AM. The effectiveness of hydrotherapy in the management of rheumatoid arthritis: a systematic review. Musculoskeletal Care 2013; 11(1):3-18.

2. Hay L, Wylie K. Towards evidence-based emergency medicine: best BETs from the Manchester Royal Infirmary. BET 4: Hydrotherapy following rotator cuff repair. EmergMed J 2001; 28(7):634-5.

3. MaddaliBongi S, Del Rosso A. How to prescribe physical exercise in rheumatology. Reumatismo 2010; 62(1):4-11.

4. Vlak T. Spondyloarthritides: principles of rehabilitation. Reumatizam 2010; 57(2):31-8.

5. Vismara L., Romei M., Galli M, Montesano A, Baccalaro G., Crivellini M, et al. Clinical implications of gait analysis in the rehabilitation of adult patients with "Prader-Willi" Syndrome: a cross-sectional comparative study ("Prader-Willi" Syndrome vs matched obese patients and healthy subjects). J NeuroengRehabil 2007; 10(4):14.

6. Davis RB, Ounpuu S, Tybursky D, Gage JR. A gait analysis data collection and reduction technique. Human MovSci 1991; 10.

7. Osorio JH, Valencia $\mathrm{MH}$. Bases para el entendimiento del proceso de la marcha humana. ArchMed (Manizales) 2013; 13(1):88-96.

8. Miyoshi T, Shirota T, Yamamoto S, Nakazawa K, Akai M. Functional roles of lower-limb joint moments while walking in water. ClinBiomech (Bristol, Avon) 2005; 20(2):194-201.

9. Schutz G, Haupenthal A, Roesler H. Estudio dinamométrico de la marcha humana en el medio acuático. Rev Digital (Buenos Aires) 2005; 10(81):15-22.

10. Barela AM, Stolf SF, Duarte M. Biomechanical characteristics of adults walking in shallow water and on land. J ElectromyogrKinesiol 2006; 16(3):250-6.

11. Romanovitch D, Vera I, Ferretti E, Costa C. Estudio comparativo de los parámetros angulares de la marcha humana en ambiente acuático y terrestre en individuos adultos jóvenes sanos. RevBrasMed Deporte 2007; 13(6): 35-47. 
Metodología para la caracterización de variables de marcha humana, mediante sistema de registro sub acuático...

12. Barela AM, Duarte M. Biomechanical characteristics of elderly individuals walking on land and in water. Brazil J ElectromyogrKinesiol 2008; 18(3):446-54.

13. Miyoshi T, Shirota T, Yamamoto SI, Nakazawa K, Akaia M. Effect of the walking speed to the lower limb joint angular displacements, joint moments and ground reaction forces during walking in water. DisabilRehabilit. 2004; 26:724-32.

14. Infantini RM, Rodrigues E. Descrição cinemática qualitativa da marcha normal dentro da água. RevFisioter UNICID 2000; 1:37-44.

15. Becker RA, Gehm F, Martínez FG, Loss JF. Análise cinemática da marcha humana em ambiente aquático Parte II: diferentes profundidades. Anais do X Congresso Brasileiro de Biomecânica; 2003 Minas Gerais, p. 111-4.

16. Gehm, F, Becker R A, Martínez FG, Loss JF. Análise cinemática da marcha humana em ambiente aquático - parte I: terra x água. In: Anais X Congresso Brasileiro de Biomecânica, 2003; Minas Gerais, p. 107-110.

17. Ervilha UF. Estudo da variação angular da articulação do joelho e do sinal eletromiográfico no domínio temporal durante a marcha humana em ambiente aquático. Dissertação. Escola de Educação Física e Esporte da Universidade de São Paulo. São Paulo; 1999.

18. Villa MA, Gutiérrez GE, Pérez MJ. Consideraciones para el análisis de la marcha humana. Técnicas de videogrametría, electromiografía y dinamometría. RevIng Biomédica 2008; 2(3)16-26.

Tabla 1. Comportamiento del tobillo en individuo femenino 1 - ángulo filtrado. Datos estadísticamente confiables: P-value< 0,05. Flexión en fase de despegue del pie

TOBILLO. Flexión en fase de despegue del pie ángulo filtrado

\begin{tabular}{cccccc}
\hline PASO & AF.P1 & AF.P2 & AF.P3 & AF.P4 & AF.P5 \\
\hline $\mathbf{1}$ & 118,03 & 110,49 & 109,27 & 116,10 & 121,33 \\
\hline $\mathbf{2}$ & 105,07 & 109,27 & 126,87 & 125,45 & 144,10 \\
\hline $\mathbf{3}$ & 116,10 & 122,68 & 137,73 & 126,87 & 149,32
\end{tabular}

Tabla 2. Comportamiento del tobillo en individuo femenino 1 - ángulo filtrado. Datos estadísticamente confiables: P-value $<0,05$. Flexión en fase de apoyo plantar

TOBILLO. Flexión en fase de apoyo plantar - ángulo filtrado

\begin{tabular}{cccccc}
\hline PASO & AF.P1 & AF.P2 & AF.P3 & AF.P4 & AF.P5 \\
\hline $\mathbf{1}$ & 89,43 & 98,63 & 90,57 & 90,57 & 88,85 \\
\hline $\mathbf{2}$ & 95,74 & 88,85 & 88,85 & 78,46 & 85,99 \\
\hline $\mathbf{3}$ & 79,63 & 84,84 & 91,72 & 77,88 & 88,28
\end{tabular}

Tabla 3. Comportamiento del tobillo en individuo femenino 2 - ángulo filtrado. Datos estadísticamente confiables: P-value<0,05. Flexión en fase de despegue del pie

TOBILLO. Flexión en fase de despegue del pie - ángulo filtrado

\begin{tabular}{cccccc}
\hline PASO & AF.P1 & AF.P2 & AF.P3 & AF.P4 & AF.P5 \\
\hline $\mathbf{1}$ & 118,03 & 118,69 & 114,20 & 125,45 & 136,05 \\
\hline $\mathbf{2}$ & 105,07 & 121,33 & 105,66 & 122,68 & 126,87 \\
\hline $\mathbf{3}$ & 116,10 & 121,33 & 142,19 & 126,87 & 128,32
\end{tabular}

Tabla 4. Comportamiento del tobillo en individuo femenino 2 - ángulo filtrado. Datos estadísticamente 
Mauricio Hernando Valencia y José Henry Osorio

confiables: P-value< 0,05. Flexión en fase de apoyo plantar

TOBILLO. Flexión en fase de apoyo plantar - ángulo filtrado

\begin{tabular}{cccccc}
\hline PASO & AF.P1 & AF.P2 & AF.P3 & AF.P4 & AF.P5 \\
\hline $\mathbf{1}$ & 89,43 & 81,95 & 84,84 & 90,57 & 84,83 \\
\hline $\mathbf{2}$ & 88,28 & 84,26 & 81,95 & 78,46 & 84,26 \\
\hline $\mathbf{3}$ & 88,85 & 91,15 & 94,01 & 78,46 & 85,41
\end{tabular}

Tabla 5. Comportamiento del tobillo en individuo masculino 1 - ángulo filtrado. Datos estadísticamente confiables: P-value<0,05. Flexión en fase de despegue del pie

TOBILLO. Flexión en fase de despegue del pie ángulo filtrado

\begin{tabular}{cccccc}
\hline PASO & AF.P1 & AF.P2 & AF.P3 & AF.P4 & AF.P5 \\
\hline $\mathbf{1}$ & 118,03 & 111,72 & 114,20 & 103,30 & 104,48 \\
\hline $\mathbf{2}$ & 115,47 & 125,45 & 124,75 & 109,88 & 118,69 \\
\hline $\mathbf{3}$ & 140,35 & 124,06 & 132,07 & 131,30 & 132,84
\end{tabular}

Tabla 6. Comportamiento del tobillo en individuo masculino 1 - ángulo filtrado. Datos estadísticamente confiables: P-value< 0,05. Flexión en fase de apoyo plantar

TOBILLO. Flexión en fase de apoyo plantar ángulo filtrado

\begin{tabular}{cccccc}
\hline PASO & AF.P1 & AF.P2 & AF.P3 & AF.P4 & AF.P5 \\
\hline $\mathbf{1}$ & 83,11 & 92,87 & 89,43 & 92,29 & 85,99 \\
\hline $\mathbf{2}$ & 85,41 & 90,00 & 74,34 & 92,87 & 71,94 \\
\hline $\mathbf{3}$ & 92,29 & 83,11 & 60,00 & 82,53 & 84,26
\end{tabular}

Tabla 7. Comportamiento del tobillo en individuo masculino 2 - ángulo filtrado. Datos estadísticamente confiables: P-value< 0,05. Flexión en fase de despegue del pie

TOBILLO. Flexión en fase de despegue del pie ángulo filtrado

\begin{tabular}{cccccc}
\hline PASO & AF.P1 & AF.P2 & AF.P3 & AF.P4 & AF.P5 \\
\hline $\mathbf{1}$ & 107,46 & 129,05 & 112,33 & 112,33 & 126,16 \\
\hline $\mathbf{2}$ & 108,66 & 122,68 & 128,32 & 111,72 & 135,23 \\
\hline $\mathbf{3}$ & 102,71 & 126,16 & 126,16 & 132,07 & 102,71
\end{tabular}

Tabla 8. Comportamiento del tobillo en individuo masculino 2 - ángulo filtrado. Datos estadísticamente confiables: P-value< 0,05. Flexión en fase de apoyo plantar

TOBILLO. Flexión en fase de apoyo plantar ángulo filtrado

\begin{tabular}{cccccc}
\hline PASO & AF.P1 & AF.P2 & AF.P3 & AF.P4 & AF.P5 \\
\hline $\mathbf{1}$ & 83,11 & 84,84 & 87,13 & 92,87 & 88,85 \\
\hline $\mathbf{2}$ & 82,53 & 74,34 & 92,87 & 91,72 & 78,46 \\
\hline $\mathbf{3}$ & 78,46 & 91,72 & 83,68 & 76,11 & 77,88
\end{tabular}

